\title{
La Simulation Réflexive Transverse : une méthode pour ancrer l'activité humaine au cœur de la performance de l'entreprise
}

Transverse Reflexive Simulation: a method to anchor human activity at the heart of a company's performance

Florence Motté et Céline Poret

\section{OpenEdition}

\section{Journals}

Édition électronique

URL : http://journals.openedition.org/activites/3123

DOI : 10.4000/activites.3123

ISSN : $1765-2723$

Éditeur

ARPACT - Association Recherches et Pratiques sur les ACTivités

Référence électronique

Florence Motté et Céline Poret, « La Simulation Réflexive Transverse : une méthode pour ancrer l'activité humaine au cœur de la performance de l'entreprise », Activités [En ligne], 15-1 | 2018, mis en ligne le 15 avril 2018, consulté le 02 mai 2019. URL : http://journals.openedition.org/activites/3123 ; DOI : 10.4000/activites.3123

Ce document a été généré automatiquement le 2 mai 2019.

\section{(c) $(1) \Theta$}

Activités est mis à disposition selon les termes de la licence Creative Commons Attribution - Pas d'Utilisation Commerciale - Pas de Modification 4.0 International. 


\section{La Simulation Réflexive Transverse : une méthode pour ancrer l'activité humaine au cœur de la performance de l'entreprise}

Transverse Reflexive Simulation: a method to anchor human activity at the heart of a company's performance

Florence Motté et Céline Poret

\section{NOTE DE L'ÉDITEUR}

Article soumis le 15/11/2017, accepté le 25/02/2018

\section{NOTE DE L'AUTEUR}

Appartenance actuelle de Céline Poret : IRSN - celine.poret@irsn.fr

\section{Introduction}

1 Depuis une vingtaine d'années, les pressions concurrentielles s'exercent dans tous les domaines d'activité. Les entreprises sont à la recherche de performance en s'appuyant encore bien souvent sur un modèle industriel alors que celles-ci tendent de plus en plus vers des activités de service (Du Tertre, 2007). Cette transformation vers de telles activités vient bouleverser la relation de service de ces entreprises impactant les métiers qui la composent, leur organisation, les outils utilisés, le nombre d'acteurs qui interviennent; le client devenant lui-même un acteur à part entière. 
2 Dans ce contexte de complexification de la relation de service, l'entreprise EDF (Électricité de France) est elle-même engagée dans cette transformation et cherche à améliorer sa performance tout en maintenant sa qualité de service. C'est dans ce cadre qu'un programme de recherche technologique en ergonomie appelé «ingénierie de la relation de service » (Motté, \& Haradji, 2010) s'est développé à la R\&D (Recherche et Développement) depuis plusieurs années. L'objectif de ce programme est d'appréhender la relation service dans sa complexité en cherchant à caractériser les différentes activités qui s'y déroulent et à concevoir pour favoriser ces activités. Son postulat consiste à affirmer que la prise en compte de l'activité humaine permet d'identifier des leviers de performance permettant à l'entreprise de se démarquer.

3 Positionner nos travaux dans un programme de recherche technologique (Theureau, 2006 ; Poizat, \& Durand, 2015) permet de leur donner une cohérence forte tout en ouvrant un champ de questionnements et d'innovations scientifiques. Celui-ci s'articule avec les préoccupations de nos directions clientes et aboutit à une alimentation réciproque entre travaux de recherche et projets opérationnels. Nous nous situons ainsi dans une dynamique de long terme destinée à participer à la transformation de l'entreprise.

4 Le socle de ce programme consiste en la mise en évidence d'une activité collective transverse cruciale pour la compréhension et l'amélioration de la relation de service alors que cette activité n'est pas visible des organisations, étant distribuée dans le temps et l'espace. Cet article présente la dernière étude que nous avons menée et dans laquelle nous avons cherché à rendre visible cette activité et à détecter des leviers de transformations pour faciliter et fluidifier la relation de service. Nous avons ainsi abouti à une méthode, la "Simulation Réflexive Transverse ", au cours de laquelle nous avons travaillé avec des conseillers pour simuler le traitement complet d'une demande client.

5 Cette simulation est le fruit du programme de recherche et des différentes études qui l'ont précédée. Pour cette raison, nous commençons cet article en présentant les fondements du programme de recherche ainsi que les connaissances et questionnements acquis progressivement quant à la caractérisation des activités mises en œuvre dans la relation de service et à la possibilité de l'appréhender du point de vue des conseillers et des clients. Ensuite nous décrivons les objectifs qui nous ont conduits à faire de la simulation, les éléments qui nous ont permis de construire la méthode, puis la méthode en elle-même (une simulation en deux parties). Après avoir présenté un certain nombre de résultats issus de la mise en œuvre de la simulation réflexive transverse, nous proposons une discussion quant à l'apport de cette méthode.

\section{Un travail ancré dans un programme de recherche en ergonomie}

\subsection{Un programme de recherche technologique sur la relation de service dans le domaine commercial}

6 Le programme de recherche dans lequel s'inscrit l'étude présentée dans cet article se déroule au sein d'EDF Recherche et Développement (R\&D) et concerne la relation de service dans le domaine commercial. Il s'articule avec les besoins des directions commerciales que ce soit pour le marché d'affaires ou le marché des clients particuliers et s'intéresse aussi bien à l'activité des conseillers travaillant dans les Centres de Relation 
Client (CRC) qu'à l'activité des clients d'EDF. Un peu plus de 6000 conseillers travaillent pour le marché d'affaires ou le marché des particuliers. Pour donner un ordre de grandeur, tous canaux confondus, les clients particuliers formulent 3,2 millions de demandes de mise en service, 1,5 millions de modification de contrat et 3,2 millions de résiliation par an, pour ne parler que de ces types de demande.

Ce programme de recherche en ergonomie s'articule autour de deux axes indissociables: un axe théorique et scientifique pour comprendre l'activité humaine et un axe technologique afin de concevoir pour cette activité. Les transformations que nous proposons visent dans un même mouvement la satisfaction du client, les conditions de réalisation du travail des acteurs et la performance de l'entreprise. Ce programme de R\&D a également pour but d'acculturer la Direction Commerce avec laquelle nous travaillons afin de l'aider à évoluer et contribuer à ces améliorations.

Notre cadre théorique s'inscrit dans le paradigme constructiviste de la cognition humaine qui considère que celle-ci n'est pas prédéterminée mais qu'elle se crée dans la situation en lien avec l'environnement (paradigme de l'enaction de Varela, 1989). Dans ce cadre, notre positionnement méthodologique consiste, en accordant un caractère central au point de vue de l'acteur, à appréhender activité individuelle et activité collective en un seul et même mouvement. En cela nous nous inscrivons dans le situationnisme méthodologique (Theureau, 2006) qui appréhende l'activité collective comme l'articulation de l'activité individuelle des différents acteurs. Par activité individuelle, nous parlons d'une activité qui intègre toujours d'autres acteurs et que Theureau (2006) qualifie «d'individuelle sociale». Ainsi le point de vue de chaque acteur n'est pas seulement individuel, il est «individuel-social» car il prend constamment en compte l'activité des autres acteurs pour agir soi-même.

\subsection{Une inscription dans la durée qui permet de progresser en termes de connaissances et de questionnements}

9 Ce travail de recherche, commencé en 2005, se développe au fur et à mesure de nouveaux questionnements et d'opportunité de travail avec nos directions commerciales, articulant constamment questionnements de recherche et questionnements opérationnels. L'objet de ce chapitre n'est pas d'en présenter tous les résultats mais d'exposer brièvement les éléments de progression de nos connaissances et de nos questionnements qui ont conduit et servi de base à l'étude présentée dans cet article. Ceux-ci sont illustrés dans la figure 1 et exposés dans la suite de cette partie. 
Figure 1 : Progression du programme de recherche en ergonomie pour la relation de service à EDF. Figure 1: Progress of the ergonomics research program for EDF's service relationship

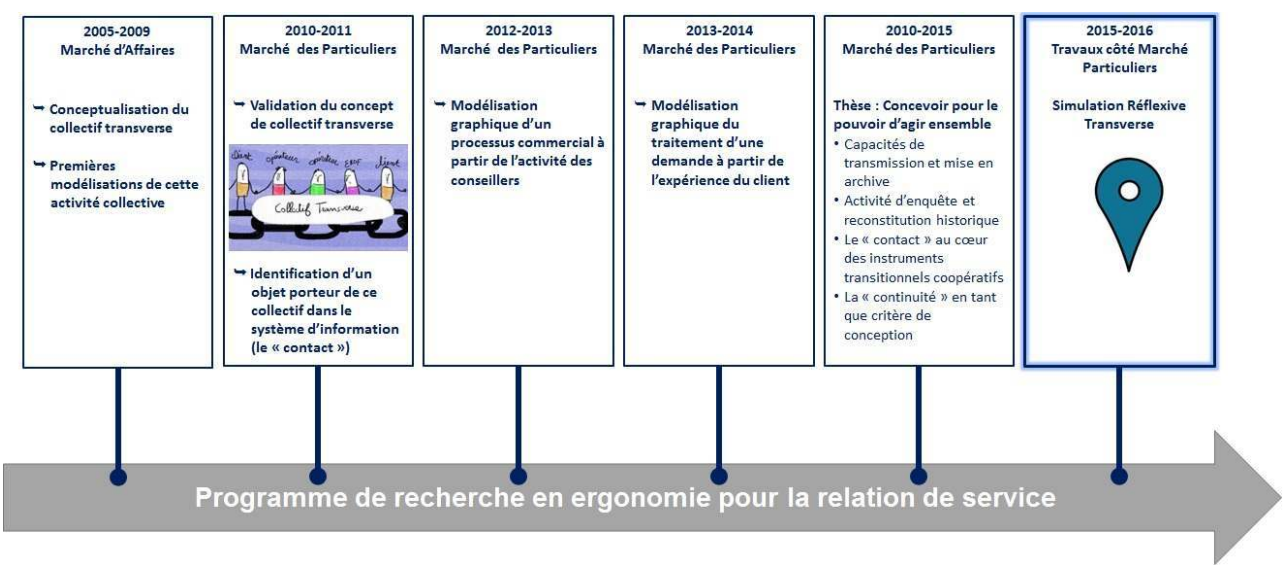

\subsubsection{Conceptualisation du collectif transverse, premières modélisations de cette activité collective (2005-2009)}

10 Les premiers travaux sur la relation de service ont été menés pour le marché d'affaires et centrés sur l'activité des conseillers qui y travaillent. Notre observatoire a d'abord concerné des vendeurs en ligne (Motté, \&, Haradji, 2007), puis des chargés de service client (contractualisation, facturation) et enfin des chargés de raccordement (lien avec les distributeurs d'énergie). Nos analyses ont ainsi porté sur l'activité réalisée au sein de chaque métier.

11 Très vite nous nous sommes rendus compte qu'il n'est pas suffisant d'analyser l'activité par type de métier pour étudier la relation de service et que celle-ci est la résultante du travail de différents acteurs qui réalisent chacun, en fonction de leur métier, une partie d'un tout aboutissant à répondre à la demande du client (Motté, \& Haradji, 2010 ; Motté, 2012). Ce résultat nous a amené à transformer notre objet d'étude pour comprendre l'activité humaine et à le porter sur la demande du client. Nous avons montré que le traitement de la demande résulte de l'articulation de l'activité individuelle des différents acteurs qui agissent en des temps et des lieux différents et nous avons qualifié cette activité collective d'activité collective transverse (Motté, \& Haradji, 2010; Motté, 2012; Poret, 2015).

Appréhender l'activité collective transverse est complexe car elle n'est pas concentrée en un même lieu et un même temps, et elle n'est pas prévisible dans la mesure où on ne sait pas quand aura lieu la suite du traitement ni généralement où (sauf en cas de portefeuille lié à un conseiller). Cette complexité est accrue par le grand nombre de clients et a fortiori par le nombre de demandes qu'ils peuvent formuler à EDF. Il n'est donc pas possible de suivre le traitement d'une seule et même demande. Modéliser l'activité collective transverse à partir d'observations d'activité des conseillers, nécessite par conséquent de reconstituer l'histoire du traitement d'un type de demande client en juxtaposant différents bouts de demandes et l'activité qui s'y rapporte (Motté 2012). Comme l'ont fait Grosjean et Lacoste $(1999$, p53) pour étudier l'activité en milieu hospitalier, nous avons dû faire des choix méthodologiques : « La plupart des études sur le travail collectif portent sur des contextes où les opérateurs sont en coprésence au sein d'un même espace ou bien sur des coopérations à distance entre des interlocuteurs bien 
identifiés. Cette relative réduction de la complexité permet d'éviter au plan méthodologique certains choix difficiles. Au contraire, les fonctionnements collectifs complexes, comportant des acteurs nombreux pris dans des situations multiples, posent un défi au chercheur, car ils obligent à des découpages et à des recompositions d'un objet dont la réalité reste à jamais insaisissable. »

\subsubsection{Validation du concept de collectif transverse, identification d'un objet porteur de ce collectif dans le système d'informations (2010-2011)}

13 Cette fois-ci, les travaux ont été menés pour le marché des particuliers et plus particulièrement la Direction des Systèmes d'Informations. Ceux-ci avaient pour objectif d'étudier l'impact de l'évolution du logiciel de gestion de la relation client (GRC) sur l'activité des conseillers. Là encore il était impossible d'observer les différentes étapes d'une même demande. Pour analyser l'activité des conseillers en front office et back office, nous avons donc construit notre recueil de données afin d'appréhender la façon dont l'activité d'un acteur se construit ici et maintenant et comment elle contribue à une activité de type collectif pour le traitement effectif de la demande (Motté, 2012). En cela nous avons fait le choix méthodologique de nous positionner dans le cadre théorique et méthodologique du cours d'action qui «permet d'appréhender la construction de l'activité collective à partir des significations construites par chacun des acteurs au cours de leurs interactions en situation » (Theureau, 2004). Ces travaux ont permis de valider le concept de collectif transverse à l'intérieur d'un même métier, celui du conseiller qui traite différents morceaux de différentes demandes mais jamais les différents morceaux d'une même demande. L'existence du collectif transverse et l'articulation entre les acteurs prennent forme par les traces que laisse chacun d'eux dans le système d'informations, notamment avec un objet du logiciel de GRC intitulé «contact » auquel est associé un champ « commentaire » (Cf. précisions en 2.2.5).

\subsubsection{Modélisation graphique d'un processus commercial à partir de l'activité des conseillers (2012-2013)}

La Direction Commerce du marché des particuliers était en train de revoir ses processus en cherchant à optimiser dans un même mouvement satisfaction client, performance économique, vente et recouvrement. Dans ce cadre, le pilote du processus SRM (Souscrire Résilier Modifier) souhaitait en réaliser une cartographie pour voir ce qu'il s'y passait. Pour l'aider dans ce travail et ancrer l'activité humaine dans cette cartographie, nous nous sommes appuyés sur nos études précédentes en prenant comme point d'entrée le traitement de la demande du client et l'activité individuelle et collective qui y est mise en œuvre et en exploitant à nouveau le recueil de données réalisé précédemment

La figure 2 illustre les principes que nous avons mis en œuvre dans cette cartographie. Considérant la demande du client, nous avons représenté son traitement à la manière d'une chronologie historique, c'est-à-dire horizontalement. Le début de notre cartographie correspondait au moment où le client rentre en interaction avec EDF et la fin correspondait au moment où la demande est entièrement terminée pour le client. Nous donnions ainsi une vision globale du traitement du début jusqu'à la fin et montrions qu'une demande de mise en service du client est bien plus large que la seule étape de souscription considérée au départ par l'équipe du processus. Cette représentation rendait visible les contributions des différents conseillers, du distributeur ainsi que du client avec 
lequel EDF a des échanges aussi bien entrants que sortants (facture notamment). Nous avons indiqué les différentes applications informatiques utilisées sans détailler les manipulations nécessaires parce que nous visions une cartographie pérenne même en cas de modifications de ces applications. Enfin, nous avons identifié les variabilités qui peuvent ralentir voire bloquer le traitement ainsi que des étapes supplémentaires de traitement en cas de détection d'anomalie.

Figure 2 : Principes d'une cartographie construite à partir de l'activité humaine. Figure 2: Principles of a mapping constructed on the basis of human activity

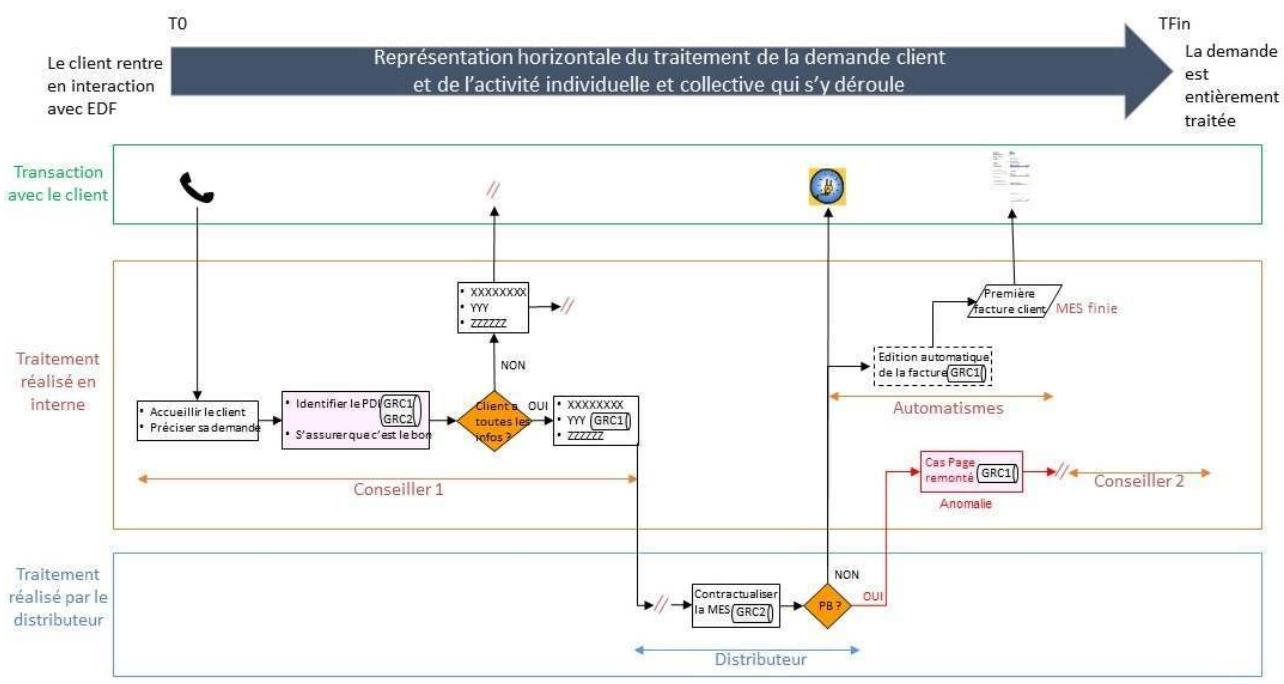

Cette représentation du processus à partir de l'activité humaine a constitué une avancée majeure pour l'équipe du processus en lui permettant de voir simplement:

- La complexité du processus avec la représentation des différents acteurs et leur activité, des différentes étapes et le temps nécessaire à une étape ;

- Les sources de variabilité (quelle que soit leur provenance) impactant le processus ainsi que les conséquences de ces variabilités.

En donnant une vision globale sur l'ensemble du traitement, la cartographie permet d'identifier les répercussions d'un dysfonctionnement mais aussi de remonter à l'origine d'un problème (Poret, \& al, 2017).

\subsubsection{Modélisation graphique du traitement d'une demande à partir de l'expérience du client (2013-2014)}

Jusqu'alors étudiée à travers le prisme de l'activité des conseillers, cette fois-ci nous avons abordé la relation de service du point de vue du client, toujours dans le cadre du marché des particuliers. Une question méthodologique s'est posée en tout premier lieu: comment constituer notre recueil de données pour reconstruire l'expérience vécue par un client au plus proche de la réalité tout au long du traitement de sa propre demande de mise en service avec EDF ? Pour cela nous avons mis au point une nouvelle méthodologie de recueil en procédant par des séances individuelles au domicile du client dont la demande était entièrement terminée et datait de moins de deux mois. Cette méthodologie incitait le client à se livrer à plusieurs narrations de plus en plus précises, avec remise en situation ou en se remémorant les interactions qu'il avait pu avoir avec tel ou tel acteur de la relation de service ou de son propre environnement. 
19 Nous avons ainsi modélisé, de façon graphique, l'histoire vécue par le client de $\mathrm{A}$ à $\mathrm{Z}$ et ses différentes interactions avec EDF ou autres acteurs (distributeur, propriétaire, famille, voisin, etc.), les informations échangées, les canaux de contacts utilisés, etc. Nous avons également fait figurer les émotions du client tout au long du traitement de la demande auxquelles nous avions pu accéder lors du recueil. Côté EDF figuraient les traces laissées dans le «contact» du logiciel de GRC auxquelles nous avions eu accès. Entrer par la demande du client et non par canal comme le faisait la direction commerciale, lui a permis d'identifier l'utilisation par le client des différents canaux pour faire avancer sa demande et son attente de cohérence entre les canaux et de fluidité dans le traitement. Ceci a mis en évidence la nécessité d'engager des chantiers pour permettre le traitement et le suivi multi-canal de la demande client.

\subsubsection{Concevoir pour le pouvoir d'agir ensemble d'un collectif transverse (thèse en ergonomie, 2010-2015)}

20 Une thèse a été réalisée (Poret, 2015) alimentant également le travail réalisé dans les projets. Elle a débuté avec l'étude pour la Directions des Systèmes d'Informations, lui permettant de recueillir ses données terrain, et elle a contribué à la cartographie du processus commercial.

21 Cette thèse a mis en évidence que l'activité collective transverse au cœur du processus consiste à articuler, à chaque étape, reconstruction historique / mise en intrigue et transmission / mise en archives. Afin de prendre la suite du traitement d'une demande qui a déjà été initiée, les conseillers ont en effet besoin d'accéder à des dimensions passées du traitement de cette demande et s'engagent pour cela dans une activité d'enquête. Le résultat de cette enquête doit leur permettre de reconstruire le cours temporel des évènements pour le traitement de cette demande ; à l'instar des historiens, ils s'engagent ainsi dans la résolution "d'intrigues " (Veyne 1971), différentes selon l'étape qu'il s'agit de traiter. Après avoir résolu l'intrigue leur permettant de comprendre l'histoire du traitement de cette demande, ils traitent l'étape et en préparent la reprise ultérieure; une de leurs préoccupations consiste en effet à articuler leur activité à celle des autres acteurs du traitement.

Pour réaliser cette articulation, les conseillers se sont appropriés le système technique et organisationnel et ont construit des «instruments transitionnels coopératifs» (Poret, 2015; Poret, \& al, 2016). Le matériau principal mobilisé dans ces instruments est constitué par les «contacts " laissés dans le logiciel de GRC et particulièrement les " commentaires " remplis par les conseillers. Les commentaires laissés par les acteurs qui ont précédé dans le traitement de la demande, servent au conseiller en charge de l'étape actuelle pour reconstruire l'histoire de ce traitement et ainsi prendre le relai. Celui-ci laisse à son tour un commentaire destiné à transmettre (ou mettre en archive) les éléments relatifs à son activité ici et maintenant pour les acteurs qui interviendront ensuite sur la demande. Ce commentaire servira alors à ceux-ci pour reconstruire l'histoire et ainsi de suite jusqu'au traitement complet de la demande. Si on adopte le plan général de la demande, ces instruments permettent ainsi une continuité du traitement de la demande malgré la discontinuité des différentes contributions caractérisant ce traitement. À partir de la mise en évidence de ces instruments, témoignant de l'existence de cette activité collective transverse antérieurement à l'intervention des chercheurs, la thèse a examiné les conditions qui complexifiaient voire rendaient impossible leur mobilisation ${ }^{1}$. Ces 
résultats ont permis de définir un critère pour la conception technique et organisationnelle: le critère de continuité. En proposant ce critère généralisable de conception l'objectif est de dépasser le stade d'ajustements locaux spécifiques à un environnement technico-organisationnel donné pour impulser un mouvement de transformation durable selon une logique transverse.

\section{La simulation réflexive transverse - Présentation de la méthode}

\subsection{Objectifs principaux}

23 À cette étape du programme de recherche, il nous semblait que l'entreprise était arrivée aux limites d'un système qui ne permet pas de rendre compte de la performance d'ensemble sur une activité morcelée, temporellement et géographiquement. Par ailleurs nous avions constaté que des effets de maximisation à la maille locale, individuelle (nombre d'appels par heure par exemple), pouvaient même aller à l'encontre d'une optimisation globale, voire de la satisfaction client. Le pilote de processus manquait de moyens, d'outils lui permettant de répondre à cette situation et de suivre la qualité de service sur l'ensemble du processus de traitement, a fortiori lorsque le client utilise des canaux différents à l'occasion d'une même demande.

Nos différentes études menées conjointement avec la Direction Commerce sur la relation de service et la mise en évidence du collectif transverse avait fini par sensibiliser nos interlocuteurs à cette notion. La prochaine étape pour nous était de la faire vivre au sein même de l'organisation et du système technique. En effet, ce collectif transverse repose sur un travail d'articulation entre les acteurs (Strauss, 1992) et un ajustement incessant. Cependant, "ce travail «supplémentaire » est souvent invisible et en tout cas largement sousestimé par rapport aux activités directement productives qu'il conditionne pourtant " (Grosjean, \& Lacoste, 1999).

Notre premier objectif était donc de révéler le collectif transverse et de créer les conditions de son développement en travaillant sur la continuité du traitement de la demande. Cette continuité concerne l'articulation entre les différents acteurs (client compris) mais aussi la visibilité des morceaux automatisés de processus. Nous souhaitions faire également un focus sur un élément essentiel porteur de la continuité dans le système d'informations, à savoir le «contact» dont l'importance avait été mise en évidence lors des études précédentes. En tant qu'ergonomes révéler l'activité a pour objectif de transformer/améliorer le processus (dans le contexte qui nous préoccupe ici) pour favoriser cette activité. Il s'agissait donc de faire un retour d'expérience sur les éléments qui favorisent ou empêchent la continuité et la fluidité du traitement d'une demande client. En perspective figurait également l'amélioration de la qualité de formation des conseillers par la mise en lumière du collectif transverse et de son déroulement en situation complexe (multi-canal, aléas du côté du client, etc.). 


\subsection{Construction de la méthode}

\subsubsection{Les questionnements de départ pour définir la méthode}

Le collectif transverse a la spécificité de se créer au fur et à mesure de l'avancée du traitement de la demande et d'être éclaté, les intervenants agissant en des lieux et à des moments différents. Pour pouvoir le révéler une solution est de mettre ces intervenants en présence (Lorino, \& Peyrolle, 2005 ; Arnoud, 2013) mais dans ce cas la complexité du travail se déroulant au fil du processus est perdue. Pour remonter le travail réel, il faut donc créer les conditions pour le simuler en simulant le traitement d'une même demande client. Pour cela, nous avons procédé à une scénarisation de situations de traitement de demande permettant d'aboutir à des cas d'étude joués et analysés avec les acteurs du collectif. Cette méthodologie a l'avantage de concrétiser un collectif de travail en rassemblant en un même lieu et un même espace-temps les acteurs de la demande, de créer des conditions de réflexivité sur leur pratique et de leur donner une vision de la globalité du traitement d'une demande qu'ils n'ont jamais. Elle permet ainsi de faire un retour sur l'ensemble du processus et de révéler l'articulation nécessaire entre les acteurs. Les équipes en CRC travaillent régulièrement sur des cas «critiques" pour montrer aux conseillers les bonnes pratiques en termes de traitement mais l'articulation entre les personnes n'est pas regardée si bien que le pilote du processus souligne l'intérêt de ce nouveau prisme d'analyse que nous lui proposons. Par ailleurs nous nous préoccupons des dimensions cognitives de l'activité, de la compréhension des «causes profondes $^{2}$ » qui amènent à traiter une demande de telle ou telle façon, notre positionnement ne vise ainsi pas le «bon traitement du cas». À l'instar de la méthode d'Analyse Approfondie d'Événement ${ }^{3}$ utilisée dans le nucléaire (Groupe d'échange ICSI " Analyse d'Événement ${ }^{4}$ », 2013 ; RON, C., 2012) notre posture consiste à révéler le travail réel, en évoquant le travail vécu au quotidien, que ce soit l'activité individuelle ou collective, et à envisager que l'organisation, la procédure, les outils, etc. peuvent empêcher le travail et être à l'origine de ces «causes profondes ». Nous ne nous situons donc pas sur le registre de «l'erreur humaine» ou du «non-respect des procédures». Repérer ces causes profondes procure des leviers de transformation et permet de viser un meilleur fonctionnement du collectif et par conséquent du processus tout en visant la qualité du traitement. Une condition sine qua non pour identifier ces causes profondes est d'instaurer un climat de confiance et de liberté d'expression.

\subsubsection{L'activité simulée : le traitement complet d'une demande client réelle}

Révéler le collectif transverse et regarder l'articulation entre les acteurs impliquait forcément de travailler sur une demande dans sa totalité. En effet, nous souhaitions montrer la répercussion d'un dysfonctionnement sur le travail des uns et des autres et sa possible « invisibilité » lorsque celui-ci intervient à la première étape et que des étapes suivantes s'amoncèlent au cours du traitement de cette même demande. Par ailleurs nous souhaitions faire intervenir le point de vue du client plus largement qu'à la suite d'une étape de traitement et accéder à son vécu sur l'ensemble de la demande.

28 La question se posait de savoir dans quel sens jouer la demande. Annoncer de but en blanc ce qui s'est passé puis remonter la demande ne permettait pas de refléter la réalité du traitement morcelé et de l'articulation entre acteurs. On ne pouvait plus alors parler de 
simulation d'activité et le risque était que cela conduise à être davantage sur le rappel des procédures que sur l'identification des causes profondes. Nous sommes donc partis sur une dynamique de traitement identique à la réalité en commençant par le début, le premier contact client.

Pour simuler l'activité des conseillers, nous avons travaillé avec deux demandes réelles entièrement traitées côté EDF et côté client. Celles-ci devaient contenir plusieurs étapes de traitement par des conseillers puisque nous travaillions sur le collectif transverse. Nous avons considéré qu'il en fallait au minimum trois. Pour être intéressantes à jouer, les demandes devaient comporter des éléments de criticité et des dysfonctionnements types pour le processus de mise en service. Le meilleur moyen pour trouver de telles demandes a consisté à puiser dans celles qui avaient fait l'objet de réclamation. Le choix s'est ensuite fait en fonction des préoccupations de la Direction Commerce (multi-canal par exemple) et des possibilités parmi les demandes récentes. Les deux demandes suivantes ont ainsi été retenues :

- Mise en service réalisée sans le client suite à une communication coupée, puis modification du conseil tarifaire (demande 1),

- Mise en service initiée sur internet puis faite par un conseiller pour cause de bug sur internet (demande 2).

30 Au cours de leur travail, les conseillers sont amenés à prendre en charge une demande client qui est soit nouvelle soit déjà en cours. Dans ce second cas ils peuvent y entrer à différents moments du traitement et c'est à eux de comprendre le besoin, ce qui a déjà été fait et ce qu'ils ont à faire. C'est pourquoi, pour chaque demande retenue pour la simulation, nous avons repéré les étapes de la demande qui étaient traitées par des conseillers et chacune d'elle a fait l'objet d'un scénario pour être joué avec les conseillers (sans leur dire qu'il s'agissait de scénarios d'une seule et même demande). Nous avions donc trois scénarios à jouer pour chacune des demandes et pour exemple ceux de la première demande étaient les suivants :

- Le client appelle mais n'est pas reconnu par le système de gestion des appels. Il doit faire des travaux de rénovation dans son nouveau logement et a constaté qu'il n'avait pas d'électricité. Il souhaite faire le nécessaire et connaît l'adresse du logement. Au moment où le conseiller dit vouloir s'engager dans le conseil tarifaire, la communication est coupée.

- Le client qui appelle dit avoir reçu une facture suite à sa demande de mise en service, il a l'électricité mais la puissance souscrite est de $6 \mathrm{kVA}$ alors qu'il souhaitait une puissance de $12 \mathrm{kVA}$. Comme ce changement nécessite l'intervention d'un technicien, le conseiller doit prendre un rendez-vous. Une fois le rendez-vous pris le client annonce que la question de la puissance ne lui a pas été posée lors de son premier appel et qu'il ne veut pas que la seconde intervention lui soit facturée.

- Le client appelle car il ne comprend pas pourquoi il a reçu des factures liées à un changement tarifaire alors qu'il n'a pas eu de confirmation du rendez-vous pris pour une augmentation de puissance (passer de 6 à $12 \mathrm{kVA}$ ) lors d'un précédent appel. Par ailleurs il ne voit pas le geste commercial qui lui avait été annoncé.

\subsubsection{Une reconstruction historique essentielle à la mise en œuvre de la simulation réflexive transverse}

31 Pour pouvoir simuler l'activité nous avons eu besoin de comprendre ce qui s'était passé sur chacune de ces demandes aux différents moments de leur traitement de $\mathrm{A}$ à $\mathrm{Z}$, aussi bien côté EDF que côté client. Il a donc fallu reconstruire l'histoire du traitement de la 
demande. Nos études précédentes ayant mis en évidence que la continuité historique de l'activité peut être reconstituée à partir des systèmes d'informations, nous avons travaillé avec un expert métier pour décortiquer les informations et les traces laissées dans les systèmes d'informations porteurs de la demande et de son traitement. Ceci a permis de comprendre la façon dont les événements avaient pu se dérouler, quelles avaient été les interventions des différents conseillers, du client, du distributeur, etc. Cet expert a ainsi procédé au travail d'enquête (Poret, 2015) auquel procèdent les conseillers pour reconstituer l'histoire d'une demande client lorsqu'ils la prennent en charge. La difficulté éprouvée par l'expert métier à faire ce travail «à tête reposée » montre la complexité de la reconstruction historique indispensable au traitement de la demande que doivent faire les conseillers au cours de leur travail quotidien, qui plus est lorsque ceux-ci sont dans le feu de l'action et avec le client au téléphone.

Outre le besoin de comprendre l'histoire des demandes, ce travail devait permettre également de reconstituer les différents états dans lesquels s'était trouvé le système d'informations à chaque étape (scénario) pour « rétablir » le contenu des écrans tels qu'ils pouvaient l'être à l'étape que l'on allait jouer, et d'avoir les éléments importants à fournir aux conseillers. En effet, les systèmes d'informations étant omniprésents dans l'activité des conseillers, il était inenvisageable de les occulter pendant la simulation. Pour créer des conditions réalistes permettant de jouer les scénarios, un travail important a donc consisté à reconstituer le contexte de travail pour chaque étape jouée. Certaines informations, comme les « commentaires » laissés par les conseillers, étant historisées il était facile de savoir lesquelles présentées au moment où se déroulait l'étape. En revanche un certain nombre de données étant saisies en remplacement d'autres, il était beaucoup plus compliqué de savoir quelles étaient les informations correspondant à l'étape et de recréer des contenus d'écrans qui soient fidèles à la situation telle qu'elle avait dû se passer... Seule la connaissance métier permettait de se prononcer à ce sujet. Ces informations ainsi identifiées ont été restituées, sous forme papier, pour pouvoir jouer les scénarios lors des sessions. La figure 3 montre la zone du commentaire associée au contact que les conseillers remplissent dans le logiciel de GRC. 
Figure 3 : Zone du « commentaire » associée au « contact » dans le logiciel de GRC.

Figure 3 : "Comment" zone associated with "contact"in CRM software

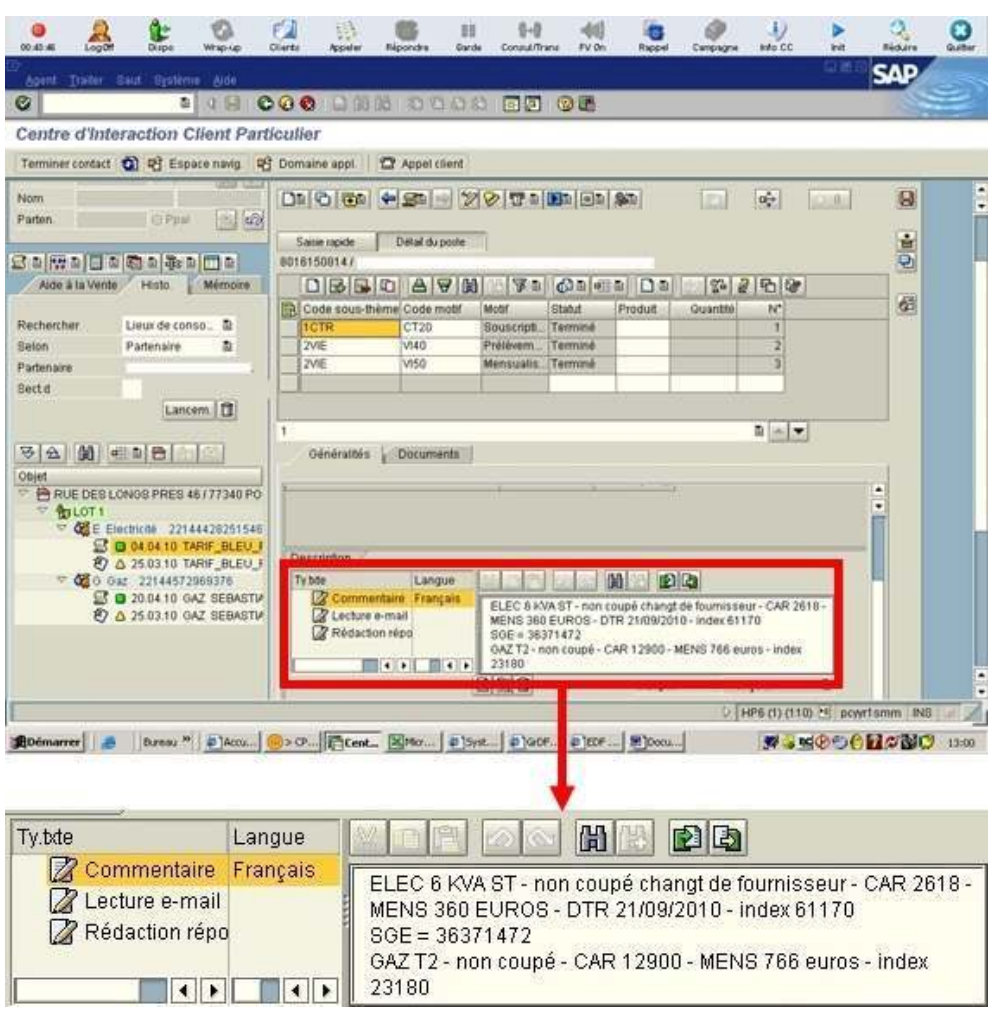

33 La figure 4 illustre le contenu d'un commentaire présenté aux conseillers lors du deuxième scénario de la demande 1 (mise en service réalisée sans le client suite à une communication coupée).

Figure 4 : «Commentaire » du deuxième scénario joué pour la demande 1. Figure 4 : "Comment" of the second scenario played out for request 1

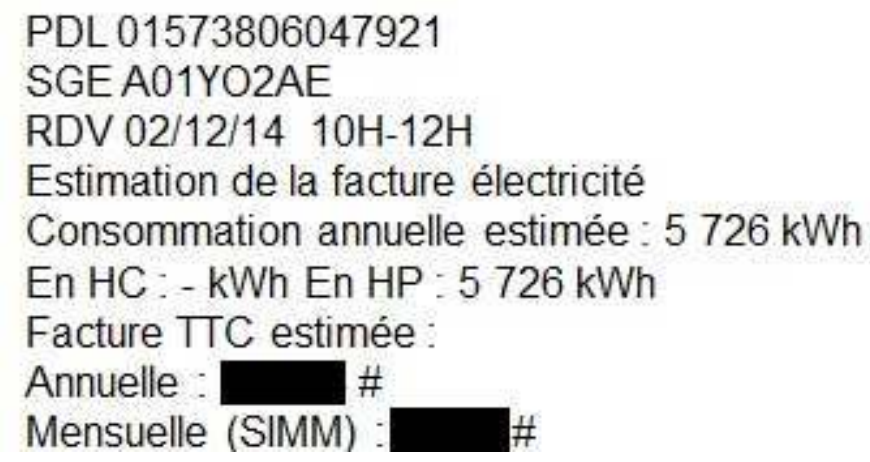

\subsubsection{Les acteurs impliqués dans les sessions}

34 L'objectif même de notre simulation concernait les conseillers, par conséquent ceux-ci étaient les premiers impliqués dans les sessions. Comme le traitement d'une demande met en jeu différents profils, aussi bien en front office qu'en back office, correspondant à des activités diverses nous souhaitions reproduire ces caractéristiques. C'est pourquoi un premier critère a été la diversité des profils des conseillers pour les sessions : mobilité (tout ce qui correspond à la résiliation ou la souscription de contrat), relation courante 
(explication de facture, règlement par $\mathrm{CB}$, modification de contrat, etc.), double compétence (mobilité + relation courante), réclamation, interlocuteur pour la mise en service sur internet par le client.

Pour pouvoir évoquer le travail réel quotidien et les éventuelles contraintes en termes organisationnels et techniques, il fallait que les conseillers aient une totale liberté d'expression. Le second critère a donc été que, pour une même session, ceux-ci viennent de CRC différents. Nous souhaitions également faire participer des conseillers qui travaillent en prestation dans des CRC spécifiques mais cela n'a malheureusement pas été possible pour des raisons réglementaires.

Pour cette même recherche de liberté d'expression et après réflexion, nous n'avons pas fait intervenir le management au cours des sessions. En effet « un lien hiérarchique entre les participants peut influencer les verbalisations. Il convient d'en tenir compte dans l'organisation des entretiens. » (Groupe d'échange ICSI « Analyse d'Événement », 2013).

Notre étude ayant des points communs avec l'Analyse Approfondie d'Événement pratiquée dans le nucléaire (Groupe d'échange ICSI « Analyse d'Événement », 2013 ; RON, C., 2012) nous nous sommes posés la question de la pertinence de faire participer les conseillers impliqués dans le traitement de la demande "jouée » en simulation. Le très grand nombre des demandes traitées ainsi que le caractère non risqué des demandes (contrairement au nucléaire) font qu'il n'y a pas de mémoire des cas traités, par conséquent il n'y avait pas d'intérêt à faire participer les conseillers qui avaient réellement traité la demande.

D'autres acteurs hors EDF sont également impliqués dans la réponse aux demandes des clients, il s'agit des distributeurs d'énergie (électricité et gaz). Comme pour les conseillers prestataires, il a été réglementairement impossible de les faire participer. Pour remédier à leur absence nous avons récupéré les actions de ces entités par l'intermédiaire des traces et des informations présentes dans les Systèmes d'informations.

Le client était incontournable dans cette simulation mais la question était de savoir s'il devait l'être physiquement lors des sessions (nous ne parlons pas là du client dans l'absolu mais du client dont la demande allait être jouée en session). Rappelons que nous visions l'évocation du travail quotidien des conseillers et non le «bon » traitement du cas, cela aurait été difficile voire impossible si le client avait été présent, d'autant plus qu'il n'aurait pas forcément compris qu'on ne cherche pas précisément à expliquer ce qui s'était passé et ce qui aurait dû se passer pour sa demande. La présence du client n'était donc pas propice aux objectifs visés pour autant son apport était indispensable. Nous avons donc cherché à reconstituer le vécu des deux clients au sujet de leur demande respective et du traitement de chacune. Pour cela nous nous sommes appuyés sur la méthode que nous avions mise au point pour notre étude concernant l'expérience vécue par le client lors de son déménagement et avons contacté chacun des deux clients concernés par les demandes jouées en simulation. Nous avons ainsi pu recueillir le point de vue de ces clients ainsi que leurs émotions pour leur propre demande.

La reconstruction de l'histoire du traitement de la demande issue du système d'information que les éléments obtenus auprès des clients nous ont permis de réaliser une modélisation graphique du traitement de chacune des deux demandes sur les mêmes principes que ceux illustrés en figure 2. Ces représentations nous ont permis de réaliser une simulation d'activité complémentaire à celle basée sur les scénarios mentionnés 
précédemment. Nous explicitons dans la partie suivante comment se sont déroulées les sessions de simulation d'activité.

\subsection{Une simulation en deux parties complémentaires}

41 Chacune des demandes jouées a fait l'objet de deux sessions avec des équipes différentes afin d'identifier des écarts potentiels; nous avons donc mené au total quatre sessions de simulation. Pour chacune des sessions sont intervenus trois conseillers, différents à chaque fois. Le tableau 1 ci-dessous récapitule ces éléments.

Tableau 1 : Diversité des profils et des CRC d'appartenance des conseillers participant aux sessions.

Table 1: Diversity of profiles and Service Relationship Centers (SRC) of the customer service advisors involved in sessions

\begin{tabular}{|c|c|c|c|c|}
\hline & \multicolumn{2}{|c|}{$\begin{array}{c}\text { Demande 1 } \\
\text { Profils }\end{array}$} & CRC & Pemande 2 \\
\hline \multirow{2}{*}{$\begin{array}{c}\text { Sessi } \\
\text { on 1 }\end{array}$} & Mobilité & A & Interlocuteur internet & CRC \\
\cline { 2 - 5 } & Double Compétence & B & Réclamation expert & D \\
\cline { 2 - 5 } & Réclamation expert & C & Relation Courante & A \\
\hline \multirow{2}{*}{$\begin{array}{c}\text { Sessi } \\
\text { on 2 }\end{array}$} & $\begin{array}{c}\text { Interlocuteur internet + Double } \\
\text { compétence récente }\end{array}$ & A & Interlocuteur internet & A \\
\cline { 2 - 5 } & Réclamation expert & D & Double compétence & C \\
\cline { 2 - 5 } & Double compétence & D & Réciamation & B \\
\hline
\end{tabular}

Chaque session a duré trois heures trois quarts environ réparties de la façon suivante :

- Introduction : présentation des participants, des travaux et du déroulement de la session (un quart d'heure).

- Partie 1 : simulation du traitement de morceaux d'une demande client (une heure trente).

- Pause (un quart d'heure).

- Partie 2 : simulation du déroulement réel du traitement d'une demande (une heure trente).

- Bilan de la session : ce que les conseillers en ont pensé (un quart d'heure).

Lors de l'introduction étaient présentés brièvement les points clés du programme de recherche, la collaboration des directions R\&D et Commerce sur ces sujets, ainsi que les objectifs de l'étude. Nous indiquions aux conseillers que nous allions travailler sur des études de cas individuellement et collectivement et que nous ne cherchions pas à identifier le «bon » traitement mais à évoquer leurs situations de travail quotidiennes à partir de ces cas. Notre but était la diversité des points de vue pour permettre d'identifier des pistes d'amélioration.

\subsubsection{Partie 1 : Simulation du traitement de morceaux d'une demande client}

$\mathrm{Au}$ cours de cette simulation chaque scénario joué articulait une séquence individuelle puis une séquence collective. Nous rappelons ici qu'il y avait trois scénarios à jouer qui correspondaient à trois étapes de la demande.

Lors de la séquence individuelle, les conseillers étaient répartis à trois coins d'une même salle pour ne pas se gêner les uns les autres. Chacun d'eux était assisté par un animateur. Pour prendre connaissance du cas à traiter chaque conseiller avait à sa disposition l'animateur qui jouait le client et des contenus d'écrans sous forme papier qui illustraient 
les systèmes d'informations pour créer des conditions réalistes et permettre un ancrage contextuel. Il était demandé au conseiller de chercher à comprendre le cas et à le traiter dans les grandes lignes en utilisant les contenus d'écrans au moment où il en avait besoin et en posant les questions qu'il poserait au client. Afin que l'animateur puisse suivre son raisonnement, il était demandé au conseiller de raisonner à voix haute et d'expliciter son raisonnement. Cette partie de traitement durait huit minutes, puis deux minutes étaient consacrées à l'écriture du « contact » que le conseiller laisserait dans le SI au sujet de ce traitement.

De retour en groupe un conseiller (volontaire) prenait la parole pour expliquer ce qu'il avait compris du cas et comment il le traiterait, les autres l'écoutaient. Ensuite l'animateur lançait la discussion collective sur les ressemblances, les différences dans le traitement $\mathrm{du}$ cas, les trucs et astuces. Il injectait également des questionnements identifiés en préparation concernant la continuité du traitement et le collectif transverse, si ces points n'avaient pas été spontanément abordés. À cet instant les conseillers confrontaient leurs pratiques individuelles et collectives en vigueur au sein de leur CRC respectif mais aussi lors du traitement des demandes. Cette partie durait quinze minutes puis cinq minutes étaient consacrées à la mise en commun des "contacts » de chacun (rassemblés et projetés au mur) et à une nouvelle confrontation sur la rédaction et le contenu des « contacts».

Le scénario 2 et le scénario 3 faisaient l'objet des mêmes séquences et à chaque fois le démarrage de la session collective se faisait sur la prise de parole volontaire d'un conseiller. Tous se sont proposés.

Cette méthode permettait d'être le plus proche possible des conditions réelles de travail des conseillers, même si la prise de connaissance et le traitement se faisait de façon macroscopique. Comme mentionné précédemment nous ne visions pas le bon traitement mais l'identification de causes profondes qui pouvaient rendre difficile le bon fonctionnement du collectif ainsi que la mise en évidence de l'articulation entre les acteurs de ce collectif.

Le déroulement des sessions a confirmé l'importance de jouer les différents cas en individuel avant de s'engager dans une discussion collective. En effet, la phase 1 permettait aux conseillers de se projeter dans l'action, de dépasser le discours, puis la phase 2 consistait en des échanges croisés riches de ces réflexions individuelles. Ces conditions associées au rôle de l'animateur ont permis à chacun(e) de s'exprimer sans retenu.

\subsubsection{Partie 2 : Simulation du déroulement réel du traitement de la demande}

Dans cette partie, la simulation se déroulait uniquement en séquence collective et concernait les échanges autour de la manière dont la demande s'était réellement déroulée. Ces échanges se faisaient sur la base de la représentation graphique sur papier du déroulement réel de la demande qui avait été construite à partir des éléments remontés par l'expert métier (reconstruction historique) ainsi que du vécu recueilli auprès du client. À l'instar des principes de représentation illustrés dans la figure 2, cette représentation était composée de 3 zones permettant de distinguer ce qui était du ressort d'EDF (zone centrale), ce qui était du ressort du distributeur (zone inférieure), et ce qui était du ressort du client (zone supérieure). 
51 Cette représentation était affichée au mur progressivement dévoilant ainsi les étapes au fur et à mesure (chaque étape occupant une feuille A3 en paysage), et était également fournie aux conseillers. Les conseillers découvraient donc le déroulement réel de la demande étape par étape. Dans cette simulation, une étape était plus fine que celle jouée dans la simulation précédente car elle pouvait correspondre à :

- Une action du conseiller, par exemple programmer un changement de puissance et l'intervention d'un technicien au compteur du client,

- Une action du client, par exemple réaliser sa mise en service sur internet,

- Une action du distributeur, comme l'envoi d'un sms ou l'intervention d'un technicien,

- Une action automatique, comme l'édition d'une facture par exemple.

Des trois étapes jouées dans la partie précédente nous passions ainsi à six étapes. Chaque étape était présentée et discutée en collectif successivement jusqu'à l'affichage complet du déroulement.

Là encore l'objectif n'était pas de trouver le «bon» traitement mais d'évoquer leur travail quotidien et de faire émerger des causes profondes ne facilitant pas leur activité : Cela leur est-il déjà arrivé ? Cela leur évoque-t-il des choses de leur quotidien ? Ont-ils des explications par rapport à ce déroulement réel? Etc. Les "contacts» et leur « commentaires » trouvés dans le SI laissés par les conseillers ayant traité la demande ou issus de traitement automatique (canal web notamment) faisaient l'objet de discussions particulières. Tout comme dans la première simulation l'animateur injectait des questionnements identifiés lors de la construction de la représentation graphique.

Dans cette partie, l'implication des conseillers était différente car la simulation consistait à les confronter à ce qui s'était réellement passé, alors que dans la première il s'agissait de ce qu'eux auraient fait. Il y avait donc complémentarité entre ces deux parties et la seconde venait stabiliser la vision du transverse abordée dans la partie 1 au travers de l'animation. Elle rendait également visible le caractère réel de la demande qui avait été jouée en « morceaux » dans la première partie. Par ailleurs elle donnait à voir le point de vue du client sur l'ensemble d'une même demande.

\subsubsection{Un rôle crucial de l'animation dans les séquences collectives}

55 Outre les conditions mises en œuvre pour favoriser la liberté d'expression (conseillers de CRC différents pour une même session, absence du management) l'animation a contribué à instaurer une atmosphère de confiance favorable aux échanges et a veillé à une expression équitable de chacun. Elle a eu un rôle crucial dans les séquences collectives car elle a permis d'accéder au raisonnement cognitif des acteurs, d'évoquer le travail réel vécu au quotidien. Lors de la première simulation, l'animation a beaucoup contribué à révéler le collectif transverse en appuyant sur les éléments d'articulation lors des échanges collectifs. La seconde simulation plaçait tout de suite les conseillers dans le collectif transverse et les amenait à commenter une demande traitée par d'autres qu'eux. Le risque était qu'ils adoptent un positionnement en retrait et en "jugement». L'animation a donc consisté à les impliquer davantage en les amenant à faire le lien avec leur quotidien.

56 L'animation était portée par les chercheurs travaillant sur le programme de recherche et l'externalité de leur regard a favorisé le contenu des échanges. Ils avaient cependant une certaine connaissance du travail des conseillers sans en être experts si bien que les conseillers étaient amenés à parler sans jargon et à lever les implicites. Une dimension 
d'explicitation importante a ainsi existé lors de ces échanges et celle-ci a été appréciée des conseillers.

Enfin l'animation a permis de creuser certains points que nous avions identifiés comme importants avant la session par rapport à l'activité des conseillers, de rebondir en direct au gré des sujets émergeant des échanges collectifs et d'aborder les possibles contraints liées au temps laissés pour réaliser une activité de front office ou back office, aux objectifs de performance, à l'organisation, aux outils, etc.

\section{Résultats liés à la mise en œuvre de la simulation réflexive transverse}

\subsection{La représentation du collectif transverse et transactionnel : une vision que n'ont jamais les conseillers}

L'objectif de révélation du collectif a été atteint et les conseillers ont apprécié d'avoir une vision globale, synthétique et directe sur le déroulement du traitement de la demande qu'ils n'avaient jamais eu l'occasion de voir et qu'a permis la représentation graphique. Ils ont apprécié de voir la répercussion que certaines de leurs actions peuvent avoir.

Le graphe c'était intéressant, on se rend compte que ça part du client. Il faut vraiment être précis, complet, dès le départ. Et la moindre petite chose, l'envoi de courrier qui finalement pour nous était au départ pas si important que ça, on se rend compte que c'est important pour le client. Ça peut éviter beaucoup de réappels » (double compétence)

«Moi je pense au premier conseiller qui ne doit même pas savoir ce que ça a engendré derrière et [...] voilà vraiment pourquoi il a rappelé (récla expert).

La vision globale et graphique qui leur est proposée diffère de la logique des outils avec lesquels ils travaillent quotidiennement et avec lesquels ils doivent faire un effort de reconstruction historique. Ils n'ont pas souvent l'occasion de se détacher de leurs outils pour travailler sur leur métier. La représentation graphique rend concret l'objet même de leur travail en matérialisant le traitement de la demande du client et son découpage en morceaux. L'affichage au mur et la place prise progressivement par la demande favorise cette prise de conscience.

C'est encore mieux, sur du papier, c'est mieux que de dire sur des pages SIMM, là, cette étape-là, voilà ce qui va se passer, on déroule... et pour le client... on a bien ça en tête... le voir comme ça on garde plus facilement que regarder dans SIMM, après on est plus vigilant (double compétence).

Cette simulation donne du sens à leur travail, en les positionnant eux et leur contribution dans un traitement global qui a un avant, un après et qui implique la contribution d'autres acteurs.

On sait à quoi s'en tenir mais c'est vraiment quand j'ai changé de file que je me suis rendue compte que les contacts étaient vraiment importants (relation courante).

61 Ils apprécient de voir distinctement les contributions et les interactions de tous les « acteurs »: les différents conseillers (" en plus on voit bien que les conseillers ce sont des conseillers différents »), le client (" donc lui (le client) pense pouvoir s'arranger avec le technicien mais il ne s'arrangera pas du tout...»), le distributeur et même les traitements automatisés qu'ils ne connaissent pas toujours bien. 
62 Avoir un retour sur le vécu du client et ses émotions tout au long du traitement de sa demande est totalement inédit et apprécié. Les conseillers découvrent le client et les suppositions qu'il peut être amené à faire.

C'est intéressant de voir les émotions du client, on se rend compte que le client va penser des choses, pas forcément réalisables. Voir le client sous ces aspects-là, c'est intéressant (internet + double compétence).

\subsection{Un ancrage du collectif transverse et transactionnel dans la pratique des conseillers}

Le fait de travailler sur des éléments porteurs de la continuité et de la fluidité du traitement a permis un ancrage du collectif transverse dans la pratique des conseillers. Utiliser un cas réel pour la simulation a fait émerger certaines problématiques qui peuvent rompre la continuité et impacter le traitement de la demande du client comme les transferts d'appels dits «sauvages » sources de quiproquos potentiels. En effet dans ces situations, l'appel du client est transféré à un autre conseiller sans les informations d'accompagnement alors que le client suppose l'inverse et donc ne répète pas tout ce qu'il a déjà pu dire. Des informations essentielles pour la suite du traitement ne sont alors pas données et la prise en charge peut s'en trouver erronée.

Le travail réalisé en session sur les " contacts ", en tant qu'objet transitionnel coopératif (Poret, 2015), a été un élément important pour ancrer le collectif transverse dans la pratique des conseillers. Si cet objet est utilisé par tous les conseillers, celui-ci ne prend son sens aujourd'hui que lors du travail en Relation Courante c'était-à-dire lorsque le conseiller est amené à davantage travailler sur la suite du traitement. Par ailleurs, la Simulation Réflexive Transverse a mis en évidence l'absence de statut clair du " contact » et de son contenu : chacun se l'approprie, le remplit et l'utilise à sa manière. Il peut être au service de la continuité dans le traitement de la demande, mais être également utilisé pour se protéger, du client ou des procédures à respecter, en détaillant particulièrement certains éléments, etc. Les "contacts» pour une même demande s'accumulent mélangeant les différents statuts, ce qui peut brouiller ou complexifier la reconstitution historique nécessaire à la compréhension de la demande. Les échanges entre pairs ont permis de montrer des points de vue tantôt homogènes, tantôt hétérogènes sur la question.

\subsection{Un espace de réflexivité et de progression pour les conseillers}

La Simulation Réflexive Transverse a eu des bénéfices directs en termes de formation pour les conseillers sur l'activité collective transverse mais aussi sur leur activité individuelle, sur le collectif métier et sur leurs interactions avec le client. Elle a fait l'objet d'une totale adhésion de leur part pour différentes raisons.

Ils ont apprécié la richesse des échanges qu'ils ont eu entre eux, leur permettant de mettre à niveau leurs connaissances, de confronter leurs pratiques, d'échanger des trucs et astuces, etc. Le fait que les échanges se fassent entre pairs sans hiérarchie différait de ce dont ils ont l'habitude, les informations étant davantage descendantes ou prescriptives. 
Les échanges qu'on a pu avoir avec mes collègues des autres agences m'ont vraiment apporté beaucoup de choses dans la mesure où, même si on est tous conseillers clientèle on n'a pas tous la même manière de fonctionner (récla expert).

Les conseillers ont également mis en évidence l'intérêt de travailler sur un cas réel qui porte une valeur incomparable à celle d'un cas fictif auquel on ne croirait pas.

J'ai apprécié que ce soit une situation réelle parce que justement on s'est rendu compte de toutes les pannes existantes dans la mise en service. À chaque fois, à chaque action on a pensé bien faire nous côté EDF, côté conseillers, et on s'est rendu compte via cet exercice des conséquences (récla expert).

8 Une grande différence remarquée a été la centration sur leur métier plutôt que sur l'outil.

C'est mieux (...) que de regarder les pages $\mathrm{SIMM}^{5}$, ce n'est pas vraiment concret. Si on veut cette étape-là voilà ce qui va se passer pour le client, dans le déroulé... Je ne connais pas tout et pourtant je ne suis pas nouvelle, je connais le métier (double compétence).

9 Le fait de passer par une séquence individuelle avant de passer en séquence collective, la liberté d'expression recherchée, le rôle de l'animation et les échanges entre pairs ont permis à certains de s'exprimer, alors qu'ils ne le font plutôt pas en temps normal, se sentant en confiance. À cet égard, une conseillère nous a fait part de sa satisfaction car elle n'osait jamais s'exprimer en temps normal.

Oui, tu as le temps d'analyser, de savoir tout, et en même temps, tu as ton truc à toi même, ce que tu vas dire, ce n'est pas quand tu écoutes tout le monde... Bon, ben, je vais dire la même chose que lui, je ne me prends pas la tête, je vais pas réfléchir (...) Ben moi, d'habitude, quand il y a du monde, tu ne m'entends même pas, je reste dans mon coin. Même ma responsable d'équipe, elle me reprochait qu'en réunion d'équipe, je ne parle pas... Là, si elle me voit, elle va me demander ce qu'il s'est passé ! (...) Parce que je sais que je n'aurais pas parlé, j'aurais dit, oui, comme elle, et puis j'aurais laissé ça comme ça, voilà...) On ne m'aurait pas entendue de la journée ! Alors que là, non! J'étais à l'aise. (Double compétence).

Un point important a été souligné car il fait souvent défaut, c'est de réfléchir et de laisser du temps aux conseillers alors qu'ils sont d'habitude constamment sous contrainte de temps. Cette «bulle temporelle» leur a permis d'avoir une réflexivité par rapport à leur propre travail, ainsi qu'au système technique et organisationnel.

D'y réfléchir, comment on peut améliorer les process, c'est déjà presque résoudre le problème. Et une notion très importante pour ce type d'atelier et pour tous les ateliers c'est laisser du temps. Le temps je pense permet la réflexion, permet de faire ressortir des choses, de mieux analyser... alors le temps, c'est compté pour tout le monde; en revanche là on l'a eu et je pense que derrière il y a du vrai bénéfice à accorder du temps (récla expert).

La Simulation Réflexive Transverse a donc eu des effets immédiats lors des sessions pour les conseillers, mais pas seulement. Elle a aussi eu des impacts au-delà des sessions ellesmêmes et des conseillers qui y ont participé. En effet, la plupart sont repartis avec la représentation graphique du déroulement complet de la demande et nous savons que cette cartographie a été exploitée par un conseiller chargé de traiter les réclamations au cours d'une réunion d'animation suite à son retour dans son Centre de Relation Client. La Simulation Réflexive Transverse a ainsi également « des effets " médiats » qui opèrent avec un décalage temporel et se diffusent en réseau auprès de bénéficiaires indirects » (Du Tertre, 2007, p. 13). 


\subsection{Une opportunité d'amélioration continue du processus}

72 Partir du travail des conseillers permet de faire émerger des éléments impactant le processus, sa performance et les conditions de réalisation du travail. Nous n'en citerons que quelques exemples parmi ceux ayant fait l'objet de présentations et de propositions d'amélioration à nos interlocuteurs commerce.

Échanger sur le travail réel, a soulevé la question de l'impact de l'évaluation de la performance sur le processus en rendant visible par exemple l'enchevêtrement d'indicateurs centrés sur la performance immédiate pouvant aller à l'encontre d'un traitement fluide et continu; la tension que doivent parfois gérer les conseillers entre productivité et satisfaction client, la recherche de performance immédiate qui peut avoir des conséquences néfastes sur la performance globale, etc. Ces résultats questionnent la pertinence de certains indicateurs notamment ceux centrés sur l'interaction «ici et maintenant » alors que la relation de service se situe sur une temporalité longue.

Certaines caractéristiques du système technique et organisationnel qui ne favorisent pas un traitement fluide et continu ont été mises en exergue. Nous avons notamment mis l'accent sur l'importance du contenu du « contact » et de sa prise de sens dans l'ensemble du processus. En termes d'apport à la conception, cela se traduit par des actions de formation pouvant s'appuyer sur ce qui a été fait durant la simulation mais aussi par des aides contextuelles qui pourraient être apportées lors de la rédaction du « commentaire».

75 Cette simulation destinée à évoquer le travail réel a montré l'impact et la densité de l'étape de souscription, traitée en mobilité, sur la mise en service et les répercussions qu'elle peut engendrer sur la suite du traitement. Cette étape impulse le processus de traitement de la demande. Là encore, ce résultat a différentes déclinaisons possibles en termes de conception : mieux adapter les outils informatiques, proposer un apprentissage sur l'ensemble de la mise en service et non juste une étape notamment pour identifier l'importance de telle information sur la suite du traitement, etc.

76 Certains principes managériaux ont été remis en question comme le fait qu'il ne soit pas possible de faire un appel sortant lors d'une coupure téléphonique (situation de plus en plus fréquente avec l'utilisation des téléphones portables) même si la transaction avait presqu'atteint son terme et qu'il suffirait de quelques minutes au conseiller pour finaliser la demande. Les répercussions seraient positives à la fois pour le client satisfait de voir sa démarche aboutir et d'être pris en considération parce qu'on le rappelle, pour le conseiller préoccupé de satisfaire le client et de mener à bien son travail, ainsi que pour l'entreprise en termes d'efficacité collective en évitant un nouvel appel du client et que celui-ci arrive sur un autre conseiller ne connaissant pas la situation, ce qui nécessiterait plus de temps. Ce résultat a conduit à revoir les pratiques existantes et à définir au niveau national les conditions de mise en œuvre du rappel client.

Cette méthode a permis également de mettre en évidence que le client est vu comme un client idéal qui ferait ce qui est prévu de faire au moment où il est sensé le faire en ayant toutes les informations nécessaires sous la main. C'est sur cette considération idéalisée du client qu'a été conçu l'outil mis à la disposition du client pour faire lui-même sa mise en service sur internet. Comme le client a ses propres besoins et ses propres contraintes qui l'éloignent du client idéal, cette considération a des conséquences sur le client qui éprouve des difficultés à l'utiliser ainsi que sur les conseillers qui se trouvent à devoir 
dépanner le client ou à prendre en charge eux-mêmes la demande. Le client que l'on voulait autonome n'en a pas les moyens et se trouve à introduire des variabilités non prévues dans le processus et son déroulement.

Bien sûr nous avons mis en avant les avantages de cette méthode soulignés par les conseillers en termes d'échanges sur leurs pratiques et de partages des différentes façons de faire méconnues voire inconnues de certains du fait des différentes initiatives possibles selon la latitude d'un centre, leur profil, etc. Ces enseignements peuvent venir enrichir à la fois les formations mais aussi des dynamiques de management.

Ces exemples permettent de voir que les évolutions possibles suite aux résultats de cette simulation constituent des transformations plus ou moins profondes. En effet, changer une politique de rappel du client peut très rapidement se faire alors que concevoir des aides contextuelles au remplissage du contact ou une modification plus importante du logiciel de GRC va nécessiter la mise en place d'un chantier de réflexion. Concernant la question des indicateurs, si certaines évolutions sont acceptées facilement par nos interlocuteurs, d'autres mettant davantage en cause le système de mesure de la performance le sont moins. Suite à nos différentes présentations, nos résultats et propositions d'évolution sont dans les mains de nos interlocuteurs commerce et des différents concepteurs concernés (processus, SI, formation, etc.). Aujourd'hui pour aller plus loin nous réfléchissons à la mise en place d'un moyen d'essai qui nous permettrait de coconstruire la relation de service avec les conseillers.

\section{Discussion}

\subsection{Un espace de construction de la continuité au service d'une ingénierie de la relation de service}

80 Dans notre programme de recherche technologique «ingénierie de la relation de service ", l'objectif de la Simulation Réflexive Transverse est double. Il s'agit, dans un même mouvement, de révéler le collectif transverse qui est encore souvent ignoré dans les organisations actuelles, impactant à la fois les conditions de travail des acteurs, la satisfaction des clients et la performance des entreprises, ainsi que de détecter des leviers de transformation, à partir d'une recherche des causes profondes, pour faciliter et fluidifier la relation de service. Pour tenir ce double objectif, nous nous sommes tournés vers les espaces de débat sur le travail (Casse, \& Caroly, 2014; Rocha, \& al, 2014 ; L'Allain, \& al, 2015), espaces de discussion (Detchessahar, \& al, 2010 ; Detchessahar, 2013) et autres espaces de confrontation de pratiques pour deux raisons. D'abord parce que les acteurs engagés dans le traitement de la demande client sont habituellement dispersés au sein d'un processus, c'est-à-dire que s'ils participent bien à la construction collective de la réponse à la demande du client ils n'interviennent toutefois ni au même moment, ni au même endroit et n'ont donc quasiment jamais l'occasion de discuter ensemble. Révéler le collectif transverse impliquait donc de les sortir de cette dispersion et de les faire se rencontrer en un même lieu et en un même temps.

81 Il s'agissait de mettre autour de la table des acteurs "qui n'avaient pas nécessairement l'occasion de se retrouver autour d'une table pour discuter de leur travail » (Petit \& Dugué, 2013, p. 217). Ensuite, parce que la recherche des causes profondes impliquait de mettre en 
discussion des éléments de connaissance acquis au fil des différentes études et d'en faire émerger de nouveaux.

Si les objectifs de ces espaces peuvent concerner aussi bien la construction de la sécurité (Casse, \& Caroly, 2014 ; Rocha, \& al, 2014 ; L'Allain, \& al, 2015) que celle de la santé au travail (Detchessahar, \& al, 2010 ; Detchessahar, 2013), l'objectif premier de la Simulation Réflexive Transverse vise celle de la continuité au cœur des organisations. Il s'agit en effet de faire prendre conscience aux acteurs impliqués dans un même processus transversal de l'interdépendance entre leurs contributions, de la coopération nécessaire pour que cette transversalité soit effective au sein des organisations, de l'expérience vécue par le client au fil du traitement de sa demande et de l'impact de leurs différentes contributions sur ce dernier. Cette prise de conscience, qui émerge progressivement de l'articulation entre les deux parties de la Simulation Réflexive Transverse, est caractérisée par la mise en visibilité de quatre éléments :

- Le processus global de traitement de la demande alors que les acteurs n'en ont généralement qu'une vision partielle puisqu'ils sont engagés dans une étape parmi d'autres de ce processus ;

- L'objet qu'ils partagent dans le cadre de leur performance collective distribuée: la demande du client et son traitement. Cette mise en visibilité est d'autant plus importante que cet objet relevant du domaine des services est par nature immatériel (Du Tertre, 2005);

- L'expérience vécue par le client au fil de ce processus qui correspond pour lui au traitement de sa demande : ses besoins, ses contraintes, ses croyances, ses émotions tout au long de ce traitement;

- Le rapport entre leur travail, la performance collective et le destinataire final de cette performance : le client. Cette prise de conscience fait dire à l'une des conseillères « qu'on voit qu'on ne se centre pas assez sur le client » alors qu'officiellement la satisfaction client est au cœur de leur travail quotidien. Les conseillers appréhendent la question de la satisfaction du client sous un angle inédit pour eux : celui du traitement de sa demande dans son ensemble, et non celui de la satisfaction immédiate à l'issue d'une interaction prise en compte par les indicateurs aujourd'hui.

\subsection{Une ingénierie inscrite dans une ontologie et une épistémologie de la complexité}

Les connaissances de l'activité humaine dans la relation de service acquises au fil des études du programme de recherche nous ont permis d'inscrire l'ingénierie de la Simulation Réflexive Transverse dans une ontologie et une épistémologie de la complexité dynamique, vivante, sociale et culturelle (Theureau, 1997). À l'instar de Detchessahar (2013) et de Van Belleghem (2016), nous pensons en effet que la discussion seule ne suffit pas et peut être vaine si l'espace de débat ou de discussion ne fait pas l'objet d'une véritable ingénierie dont l'objectif est notamment de ramener ou de "convoquer" (Van Belleghem, ibid) le travail réel. Or, le fait de réunir autour d'une même table des acteurs qui sont habituellement dispersés dans le temps et l'espace constitue une forme de simplification qu'il s'agit de prendre en compte. En concevant la Simulation Réflexive Transverse à partir des résultats d'études antérieures relatives au travail des conseillers en situation naturelle, nous cherchions à simuler des phénomènes cognitifs (Theureau, ibid) présents lors de ces situations naturelles (enquête historique, transmission et mise en archive, etc.). L'objectif était de reproduire ces phénomènes en 
simulant les spécificités des situations naturelles, à savoir leur distribution temporelle et spatiale, à laquelle correspond une distribution technique et organisationnelle. Placer les différents acteurs d'un même processus autour d'une table, en un même lieu et en un même espace ne permet pas d'accéder précisément à cette connaissance, notamment pour ce qui est de la dimension technique.

Pour simuler les phénomènes cognitifs se déroulant lors de situations naturelles, il faut avoir une connaissance de l'activité collective au fil du processus, dans sa distribution temporelle et spatiale, et comprendre ce que coopérer de manière transverse à l'organisation implique en termes d'activité à chaque étape pour chacun des acteurs engagés. Ce sont les études réalisées au fil du programme de recherche qui nous ont permis d'acquérir les connaissances techniques et organisationnelles nécessaires, et notamment de mettre en évidence le rôle crucial des "contacts", l'existence d'instruments transitionnels coopératifs (Poret, 2015 ; Poret, \& al, 2016) à chaque étape permettant une certaine continuité de l'activité collective malgré la discontinuité résultant des différentes expériences singulières. Ainsi, si nous avons travaillé avec les conseillers sur les "contacts", c'est bien grâce à cette connaissance de l'activité individuelle et collective au fil du processus. C'est également cette connaissance qui a permis d'assurer une animation dont le rôle a été crucial pour atteindre le double objectif de la Simulation Réflexive Transverse et de construire l'ensemble du support nécessaire à la simulation.

\subsection{Un espace réflexif comme moteur de transformations}

La Simulation Réflexive Transverse a été conçue comme un espace de construction de la continuité, élément crucial d'une relation de service de qualité. La réflexivité qui est au cœur de tels espaces a permis non seulement d'atteindre les objectifs fixés mais aussi d'aller plus loin en provoquant des développements individuels et collectifs qui permettent des transformations à différents niveaux.

Ainsi, à l'instar de ce que décrit Daniellou (2007) relativement aux résultats de la simulation des situations de travail en ergonomie, la Simulation Réflexive Transverse permet des bénéfices formatifs et développementaux grâce à la confrontation des mondes qu'elle favorise. En effet, le fait d'échanger entre pairs de différents profils, de différents Centres de Relation Client sur la base de la confrontation des pratiques individuelles a créé un espace de «plurilinguisme professionnel » (Duboscq, \& Clot, 2010, p. 264), à l'origine d'une élaboration non seulement personnelle en permettant à chacun d'avoir une posture réflexive relativement à sa propre pratique mais également interpersonnelle. La Simulation Réflexive Transverse permet en cela des développements au niveau de l'activité individuelle, au niveau de l'activité collective métier (entre pairs), et au niveau de l'activité collective transverse. Elle constitue en cela un espace réflexif qui permet de développer à la fois les individus, les collectifs et l'organisation (Mollo, \& Nascimento, 2013).

De plus, la Simulation Réflexive Transverse, par son objectif de transformation, permet de faire remonter des éléments relatifs aux facteurs qui complexifient voire empêchent la réalisation de l'activité collective transverse ainsi que l'activité du client, impactant la continuité nécessaire à une relation de service de qualité. Ces éléments sont pris en compte par les conseillers lorsqu'ils retournent à leur poste habituel, dans la distribution temporelle et spatiale caractérisant les processus transversaux, et diffusés plus largement 
au sein de leurs Centres de Relation Client. Ils sont également discutés entre les chercheurs et les directions opérationnelles pour proposer des chantiers de transformation.

\section{Conclusion}

Par l'activité collective transverse qu'elle permet de révéler et de discuter, la méthodologie que nous avons présentée dans cet article - la Simulation Réflexive Transverse - constitue une simulation de processus. Dans la course à l'optimisation des processus, il existe actuellement des propositions pour les simuler et ainsi anticiper l'impact de transformations apportées dans le cadre d'une réingénierie de processus sur la performance globale (Kim, \& al, 2005 ; Doomun, \& al, 2008; Shim, \& al, 2010). Ces simulations sont numériques et modélisent le processus uniquement sous le scope d'un flux d'informations. La Simulation Réflexive Transverse s'en distingue en adoptant un point de vue inédit: celui de la complexité de l'activité humaine. Cela entraîne deux différences majeures avec les simulations numériques: il s'agit d'une simulation participative centrée sur le travail et le processus y est modélisé comme une coordination d'activités individuelles qui implique une coopération intra- et intermétiers. Ainsi, intégrée à la vie courante de l'organisation, la Simulation Réflexive Transverse - par sa dimension participative - peut entrâner des développements sans cesse renouvelés de l'activité individuelle et collective à l'origine d'une amélioration continue de la performance transversale. Mobilisée spécifiquement dans le cadre d'une réingénierie de processus, la Simulation Réflexive Transverse peut permettre d'identifier l'impact des transformations envisagées sur le travail et sur la coopération intra et intermétiers qui est au cœur de cette performance. Il s'agit là d'un levier de performance basé sur l'activité humaine particulièrement utile dans un contexte d'automatisation des processus (Business Process Automation) puisque la Simulation Réflexive Transverse permet d'anticiper les impacts de l'automatisation sur l'activité, dans ses dimensions individuelles et collectives, et donc sur la performance dans son ensemble.

Par ailleurs, la Simulation Réflexive enrichit le programme de recherche technologique et participe de la transformation durable de l'entreprise qui a été initiée depuis plusieurs années par notre programme de recherche. La Simulation Réflexive Transverse montre que cette transformation durable implique un mode différent de rapport au travail et au temps: passer d'une logique de performance individuelle pilotée à court-terme et potentiellement contre-productive à une logique de performance collective qui implique notamment de laisser du temps pour parler du travail. Il s'agit de permettre de prendre du temps sur la production directe des conseillers afin d'ouvrir des espaces de construction de la continuité, de progression des compétences, de développement des individus, des collectifs et des organisations et ainsi permettre une performance de l'entreprise sur le long terme (Du Tertre, 2013). En cela, la Simulation Réflexive Transverse et le programme de recherche dans lequel elle s'inscrit participent d'une transformation durable de la conceptualisation de la performance que nous proposons de fonder à partir de l'activité humaine (Poret, \& al, 2017).

Le critère de continuité et la Simulation Réflexive Transverse définis dans le cadre d'une ingénierie de la relation de service sont valables dans d'autres domaines dont la caractéristique commune est d'impliquer une production collective qui intègre différents acteurs de même métier, de métiers différents voire d'entreprises différentes dans le 
cadre d'une coopération distribuée dans le temps et l'espace. C'est le cas pour des biens ou des services dont la production implique une coopération aussi bien intraorganisationnelle dans le cadre de processus qu'inter-organisationnelle dans le cadre de Supply Chains ou réseaux inter-organisationnels. Aujourd'hui, à la suite du recentrage des entreprises sur leur cœur de métier et de l'externalisation des autres activités au sein de réseaux de partenaires, "une bonne partie de l'activité industrielle moderne (à l'inclusion des services) appelle des chaines de sous-traitance pouvant aller jusqu'à huit ou dix maillons successifs" (Ségrestin, 2004, p. 134). Cette configuration de production collective distribuée place la question de la continuité au cœur des préoccupations du Supply Chain Management (Coursaget, \& al, 2014).

La pertinence de la Simulation Réflexive Transverse pour traiter de ces questions communes à de nombreux domaines, telles que la simulation de processus et le Supply Chain Management, ouvre des perspectives prometteuses en termes de recherche.

\section{BIBLIOGRAPHIE}

Arnoud, J. (2013). Conception organisationnelle : pour des interventions capacitantes. . Paris : Thèse de doctorat du Conservatoire National des Arts et Métiers (CNAM).

Casse, C., \& Caroly, S. (2014). Concevoir un dispositif de Retour d'Expérience intégré à l'activité collective. 49ème Congrès de la SELF (La Rochelle), 327-335.

Coursaget, A., \& Tête, F. (2014). La continuité de la Supply Chain. Sécurité et stratégie, 3(18), 45-52.

Daniellou, F. (2007). Des fonctions de la simulation des situations de travail en ergonomie.

Activités, 4(2), 77-83.

Detchessahar, M. (2013). Faire face aux risques psycho-sociaux : quelques éléments d'un management par la discussion. Négocitaions, 1(19), 57-80.

Detchessahar, M., Devigne, M., \& Stimec, A. (2010). Les modes de régulation du travail et leurs effets sur la santé des salariés : deux établissements d'accueil des personnes âgées en quête de management. Finance Contrôle Stratégie, 13(4), 39-74.

Doomun, R., \& Vunka Jungum, N. (2008). Business Process modelling, simulation and reengineering: call centres. Business Process Management Journal, 14(6), 838-848.

Du Tertre, C. (2005). Services immatériels et relationnels : intensité du travail et santé. Activités, 2 (1), 37-49.

Du Tertre, C. (2007). "Modèle industriel" et "modèle serviciel" de performance. 17th International RESER Conference, Tampere (Finland), http://www.iddlab.org/data/sources/users/5/docs/ modele_industriel_et_modele_serviciel_de_performance.pdf.

Du Tertre, C. (2013). Economie servicielle et travail : contribution théorique au développement “d'une économie de la coopération". Travailler (29), 29-64. 
Duboscq, J., \& Clot, Y. (2010). L'autoconfrontation croisée comme instrument d'action au travers du dialogue : objets, adresses et gestes renouvelés. Revue d'anthropologie des connaissances, 4(2), 255-286.

Grosjean, M., \& Lacoste, M. (1999). Communication et intelligence collective. Le travail à l'hôpital. Paris : PUF, Le Travail Humain.

Groupe d'échange ICSI “Analyse d'Evènement”. (2013). Numéro Numéro 2014-04 des Cahiers de la Sécurité Industrielle, Institut pour une Culture de Sécurité Industrielle. Toulouse : ISSN 2100-3874.

Haradji, Y., \& Faveaux, L. (2006). Evolution de notre pratique de conception (1985-2005) : modéliser pour mieux coopérer à partir des critères d'utilité, d'utilisabilité. Activités, 3(1), 67-98.

Haué, J.-B. (2004). Intégrer les aspects situés de l'activité dans une ingénierie cognitive centrée sur la situation d'utilisation. @ctivités, 1(2), pp. 170-194. www.activites.org.

Kim, J.-I., Yim, D.-S., Choi, J.-S., \& Kim, K.-C. (2005). A methodology for integrating business process and simulation for business process redesign. Asian Journal on Quality, 6(1), 74-97.

L'Allain, C., Caroly, S., \& Drais, E. (2015). Les espaces de débat sur le travail : des ressources pour la prévention des risques dans un contexte d'innovations et d'incertitudes. Actes du 50ème Congrès de la Société d'Ergonomie de Langue Française (SELF), 316-322.

Lorino, P., \& Peyrolle, J.-C. (2005). Démarche pragmatiste et mise en processus dans les situations de gestion. Dans R. Teulier, \& P. Lorino, Entre connaissance et organisation : l'activité collective (pp. 220-229). Paris : La Découverte.

Mollo, V., \& Nascimento, A. (2013). Pratiques réflexives et développement des individus, des collectifs et des organisations. Dans P. Falzon, Ergonomie constructive (pp. 207-221).

Motté, F. (2012). Le collectif transverse : un nouveau concept pour transformer l'activité. Actes du 47ème Congrès de la Société d'Ergonomie de Langue Française (SELF), 102-108.

Motté, F., \& Haradji, Y. (2007). Le vendeur en ligne dans la relation de service. Dans M. Zouinar, G. Valléry, \& M.-C. Le Port, Ergonomie des produits et des services. XXXXIIe Congrès de la SELF, SaintMalo (pp. 257-265). Octarès.

Motté, F., \& Haradji, Y. (2010). Construire la relation de service en considérant l'activité humaine dans ses dimensions individuelles et collectives. Dans G. Valléry, M.-C. Le Port, \& M. Zouinar, Ergonomie, conception de produits et services médiatisés. Paris : PUF.

Motté, F., \& Lambert, I. (2008). S'appuyer sur l'analyse de l'activité pour concevoir une relation de service de qualité. Dans P. Negroni, \& Y. Haradji, Ergonomie et conception, 43ème Congrès de la SELF, Ajaccio (pp. 44-52). ANACT.

Petit, J., \& Dugué, B. (2013). Structurer l'organisation pour développer le pouvoir d'agir : le rôle possible de l'intervention en ergonomie. Activités, 10(2), 210-228.

Poizat, G., \& Durand, M. (2015). Analyse de l'activité humaine et éducation des adultes : faits et valeurs dans un programme de recherche finalisée. Revue française de pédagogie(190), 51-62.

Poret, C. (2011). L'articulation invisible des acteurs de la relation de service : une condition indispensable pour répondre à la demande du client. Séminaire Ergo'IdF (Cerf M., Motté F., Folcher V.), Entre continuité de la relation de service et discontinuité des activités : quelles contributions de l'ergonomie?, Paris, 18 octobre.

Poret, C. (2015). Concevoir pour le Pouvoir d'agir ensemble d'un collectif transverse. Le cas de la relation de service dans le domaine commercial. Thèse de doctorat - Université Paris VIII. 
Poret, C., Folcher, V., Motté, F., \& Haradji, Y. (2016). Concevoir pour le Pouvoir d'agir ensemble au sein des organisations : le cas d'un processus commercial. Activités, 13(2).

Poret, C., Motté, F., \& Leget, L. (2017). L'ergonomie au service de la performance commerciale de l'entreprise ? . 52ème Congrès de la SELF - Présent et Futur de l'Ergonomie (Toulouse, 20-22 septembre), 85-90.

Rocha, R., Mollo, V., \& Daniellou, F. (2014). Work debate spaces: A tool for developing a participatory safety management. Applied Ergonomics, 46, 107-114.

Ron, C. (2012). Analyse Approfondie d'Evènements (Recherche des causes profondes) ou Comment éviter la théorie de la pomme pourrie. Présentation powerpoint - www.asn.fr.

Salembier, P., \& Zouinar, M. (2004). Intelligibilité mutuelle et contexte partagé. Inspirations conceptuelles et réductions technologiques. Activités, 1(2), 64-85.

Segrestin, D. (2004). Les chentiers du manager. L'innovation en entreprise : où en sommes-nous? Comment piloter les changements et les maîtriser? Paris : Armand Colin.

Shim, S. J., \& Kumar, A. (2010). Simulation for emergency care process reengineering in hospitals. Business Process Management Journal, 16(5), 795-805.

Strauss, A. (1992). La trame de la négociation. Paris : L'Harmattan.

Theureau, J. (1997). L'utilisation des simulateurs de salle de contrôle de réacteur nucléaire et de cockpit d'avion à des fins autres que de formation : présentation et discussion des tendances actuelles. Dans P. Béguin, \& A. Weill-Fassina, La simulation en ergonomie : connaître, agir et interagir (pp. 113-136). Toulouse : Octarès.

Theureau, J. (2004). Le cours d'action: Méthode élémentaire. Toulouse : Octarès.

Theureau, J. (2006). Le cours d'action : méthode développée. Toulouse: Octarès.

Van Belleghem, L. (2016). Eliciting activity: a method of analysis at the service of discussion. Le Travail Humain, 79(3), 285-306.

Varela, F. (1989). Invitation aux sciences cognitives. Paris : Editions du Seuil.

Veyne, P. (1971). Comment on écrit l'histoire. Paris : Editions du Seuil.

\section{NOTES}

1. Par exemple, la thèse a montré que certains indicateurs de performance, relatifs à chaque étape, pouvaient empêcher la mobilisation des instruments transitionnels coopératifs au service du traitement global.

2. "État du système sociotechnique qui a créé les conditions nécessaires à la survenue d'un événement. (...) on considère qu'une cause profonde peut relever des dimensions organisationnelles, managériales et/ou facteurs humains» (Groupe d'échange ICSI "Analyse d'Événement ", 2013, p. 18).

3. Il s'agit d'une «situation particulière de prise en compte des facteurs humains et organisationnels» (ibid, p. 11)

4. «L'Institut pour une culture de sécurité industrielle (Icsi) est une association loi 1901 dont la vocation est de faire progresser la culture de sécurité en France. Il est né en 2003 à l'initiative de huit partenaires fondateurs (Airbus, ArcelorMittal, CNRS, Communauté d'agglomération du Grand Toulouse, EDF, Institut National Polytechnique de Toulouse, Région Midi-Pyrénées et Total) qui ont été rapidement rejoints par d'autres industriels de branches diverses, des Instituts 
spécialisés, des Écoles et Universités, des acteurs de la société civile (associations de maires, organisations syndicales, organisations non gouvernementales).» (Ibid, p. 3)

5. Il s'agit du logiciel de GRC qu'utilisent quotidiennement les conseillers.

\section{RÉSUMÉS}

Cet article présente la genèse et la mise en œuvre d'une méthode qui consiste à simuler l'activité de traitement d'une demande client dans le domaine de la relation de service. L'objectif de cette simulation était double : révéler une activité collective qui se déroule de façon distribuée dans le temps et dans l'espace et identifier des leviers de transformation pour faciliter et fluidifier la relation de service. Cette simulation, que nous avons nommée «Simulation Réflexive Transverse » convoque le travail par le biais des connaissances acquises sur l'activité humaine dans ses différentes dimensions au travers d'un programme de recherche technologique existant au sein de la R\&D (Recherche et Développement) d'une grande entreprise. Elle permet ainsi de travailler sur la continuité, élément crucial de la qualité de service et de la satisfaction client, en ouvrant des espaces de construction de cette continuité. En favorisant le développement des individus, des collectifs et des organisations, la Simulation Réflexive Transverse contribue à la performance de l'entreprise sur le long terme.

This article presents the genesis and implementation of a method that consisted in simulating the activity of processing a customer request in the field of the service relationship. The purpose of this simulation was twofold: to reveal a collective activity that unfolds in a distributed manner in time and space and to identify the levers of transformation to facilitate and to make the service relationship more fluid. This simulation, known as "Transverse Reflexive Simulation", elicits the work through the knowledge acquired on human activity in its various dimensions in a large company's existing R\&D (Research and Development) program. It allows one to work on continuity - element crucial to quality of service and customer satisfaction - by opening spaces in which to construct this continuity. By promoting the development of individuals, collectives and organizations, Transverse Reflexive Simulation contributes to a company's long-term performance.

\section{INDEX}

Keywords : simulation of human activity, cross-functional collective activity, service relationship, design, modelling

Mots-clés : simulation d'activité humaine, activité collective transverse, relation de service, conception, modélisation

\section{AUTEURS}

\section{FLORENCE MOTTÉ}

EDF R\&D

florence.motte@edf.fr 


\section{CÉLINE PORET}

Université Paris 8

poret_celine@yahoo.fr 